
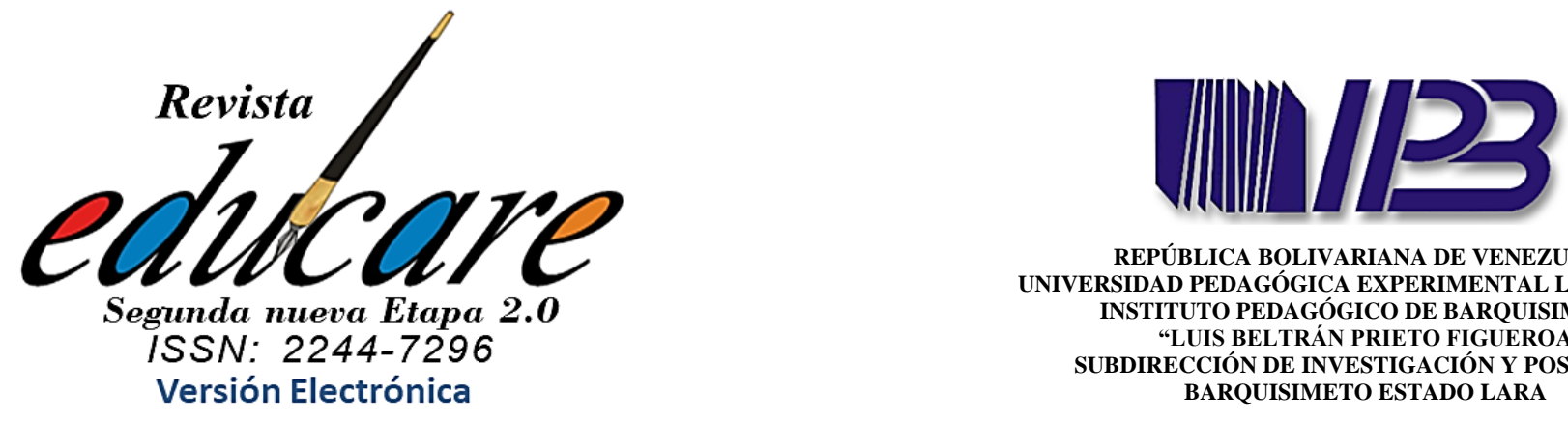

REPÚBLICA BOLIVARIANA DE VENEZUELA UNIVERSIDAD PEDAGÓGICA EXPERIMENTAL LIBERTADOR INSTITUTO PEDAGÓGICO DE BARQUISIMETO "LUIS BELTRÁN PRIETO FIGUEROA

SUBDIRECCIÓN DE INVESTIGACIÓN Y POSGRADO BARQUISIMETO ESTADO LARA

Volumen 24 № 1 Enero-Abril 2020

(291-302)

María Yessenia Macías Vera* ORCID:http://orcid.org/0000-0001 9728-5921

Carlos Eduardo Corral Mendoza** ORCID: http://orcid.org/0000-0001-9728-5921

Ligia María Izurieta Rubira***

ORCID: http://orcid.org/0000-0001-9728-5921

\author{
UNIVERSIDAD LAICA "ELOY \\ ALFARO \\ ECUADOR
}

*Licenciada en Administración de Empresas Turísticas y Hoteleras, en la Universidad Laica "Eloy Alfaro" de Manabí. Magister en Marketing de Destinos y Productos Turísticos en la ESPOL.E-mail.mariay.macias@uleam.edu.ec

** Ingeniero en Contabilidad y Auditoría y además el título de Contador Público Autorizado, en la Universidad Técnica de Manabí. Magister en Dirección y Asesoramiento Financiero, en la Universidad de la Rioja España UNIR.

edu_corral@hotmail.com Licenciada en Administración de Empresas Turísticas y Hoteleras, en la Universidad Laica "Eloy Alfaro" de Manabí. Magister en Marketing de Destinos y Productos Turísticos en la ESPOL. Docente de la Unidad Educativa Fiscal "Pedro Balda Cucalón";

ligiaizurieta@hotmail.com

\section{EDUCACIÓN AMBIENTAL Y TURISMO SOSTENIBLE: APORTES PARA LA CIUDAD DE MANTA ECUADOR}

\author{
ENVIRONMENTAL EDUCATION AND SUSTAINABLE \\ TOURISM: CONTRIBUTIONS TO THE CITY OF \\ MANTA ECUADOR
}

Recibido:

15-10-2019

Aceptado:

22-02-2020 

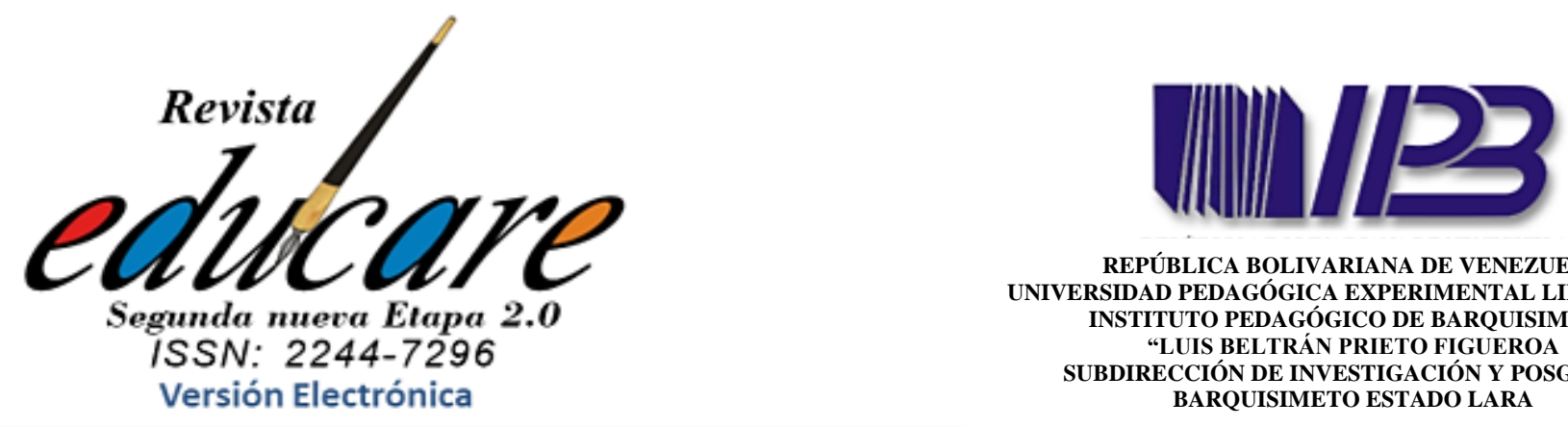

REPÚBLICA BOLIVARIANA DE VENEZUELA UNIVERSIDAD PEDAGÓGICA EXPERIMENTAL LIBERTADOR INSTITUTO PEDAGÓGICO DE BARQUISIMETO "LUIS BELTRÁN PRIETO FIGUEROA SUBDIRECCIÓN DE INVESTIGACIÓN Y POSGRADO BARQUISIMETO ESTADO LARA

\section{EDUCACIÓN AMBIENTAL Y TURISMO SOSTENIBLE: APORTES PARA LA CIUDAD DE MANTA ECUADOR}

\author{
ENVIRONMENTAL \\ EDUCATION AND \\ SUSTAINABLE TOURISM: \\ CONTRIBUTIONS TO THE \\ CITY OF MANTA ECUADOR
}

\section{Resumen}

El presente artículo, se suscribe en la modalidad de ensayo, el cual tiene como propósito realizar una contribución crítica y reflexiva en torno a la educación ambiental como herramienta estratégica para fomentar el turismo sostenible en la ciudad de Manta Ecuador, dicho recorrido discursivo inicia con una mirada hacia los aportes y preceptos que han realizado los organismos internacionales en torno a la temática, de allí que se aprecia que el turismo sostenible es crucial para el desarrollo, no solo porque encierra el potencial de contribuir al crecimiento económico, sino que también puede ejercer una influencia positiva en las comunidades locales; preservar el patrimonio, la biodiversidad y los recursos naturales. En este sentido se asume que la educación ambiental es una herramienta para mejorar las relaciones de los seres humanos con su medio y, por lo tanto, debe incidir en la prevención y resolución de problemas ambientales. Como reflexión, es imperativo que se asuma un currículo educativo contextualizado, que incluya la educación ambiental como la herramienta para impulsar el turismo sostenible, donde los docentes desarrollen estrategias con mayores niveles de concreción y con una postura socio crítica más cónsona con las demandas sociales y ambientales de la localidad.

Descriptores: educación ambiental; turismo sustentable; ciudad de Manta

\begin{abstract}
This article, is part of the trial modality, which aims to make a critical and reflective contribution around environmental education as a strategic tool to promote sustainable tourism in the city of Manta Ecuador, this discursive journey begins with a look at the contributions and precepts that international bodies have made around the subject, hence it is appreciated that sustainable tourism is crucial for development, not only because it holds the potential to contribute to economic growth, but can also have a positive influence on local communities; preserve heritage, biodiversity and natural resources. In this sense it is assumed that environmental education is a tool to improve human relations with their environment and should therefore influence the prevention and resolution of environmental problems. As a reflection, it is imperative that a contextualized educational curriculum be assumed, including environmental education as the tool to promote sustainable tourism, where teachers develop strategies with higher levels of concreteness and with a more consosient socio-critical stance with the social and environmental demands of the locality.
\end{abstract}

Keywords: environmental education; sustainable tourism; Manta City. 


\section{PRELUDIO:}

\section{UNA MIRADA DESDE LOS ORGANISMOS INTERNACIONALES}

Han transcurrido 20 años, desde que el parlamento Latinoamericano y la Comisión Permanente de Turismo resolvieron promover en los estados miembros la inclusión en el currículo educativo una formación para el turismo desde el nivel de educación inicial primario con el propósito de crear una actitud turística nacional latinoamericana, orientada al conocimiento de las bellezas naturales, la preservación del medio ambiente, a la promoción de los países en materia turística y a la mejor atención del visitante.

Asimismo la comisión internacional de la UNESCO de Educación Científica, Tecnológica y Ambiental en su asamblea del año 2000 centraron su tarea en el turismo y como consecuencia de ello, desarrollaron programas de trabajo a largo plazo que extendería hasta el 2030 para el turismo sustentable, a la que se les unió la Convención para la Diversidad Biológica proponiendo un programas de turismo de las instituciones financieras bilaterales y multilaterales colocándolo en lo alto de sus listas de prioridades.

Se puede notar que con el paso del tiempo el turismo ha crecido de manera inmensurable, y esto ha traído como consecuencia una repercusión bastante negativa en el medio ambiente y a las localidades de acogida, por lo que desde la misma UNESCO en el año 2017 hace un llamado a todas las naciones a sumar esfuerzo y prestar más atención al turismo sostenible; el cual es definido por la Organización Mundial del Turismo (OMT) como: "El turismo que tiene plenamente en cuenta las repercusiones actuales y futuras, económicas, sociales y medioambientales para satisfacer las necesidades de los visitantes, de la industria, del entorno y de las comunidades anfitrionas."(OMT 2005 p 11). De igual forma Troncoso (2012) lo define como un modelo de desarrollo económico concebido para mejorar la calidad de vida de la comunidad receptora, para facilitar al visitante una experiencia de alta eficacia y mantener la calidad del medio ambiente, del que tanto la comunidad anfitriona como los visitantes dependen.

El turismo sostenible de acuerdo a la Red de Periodistas por el Desarrollo Sostenible (RPDS) (2018) es una industria que busca mitigar su impacto sobre el medio ambiente y las comunidades cercanas en donde se realiza esta actividad, el cual genera nuevos ingresos económicos para el territorio, creando nuevas oportunidades laborales y garantizando la protección y conservación de los ecosistemas locales; es una forma de impulsar un turismo 
responsable que es ecológicamente y culturalmente amigable y que al implementarlo también se involucra a todas las partes (comunidades, empresas y turistas), creando actividades que tienen un impacto mínimo sobre la naturaleza, aprendiendo a proteger la biodiversidad de la zona y aprovechar los recursos naturales que se pueden encontrar en el sector, creando de esta forma una experiencia totalmente diferente y gratificante.

Dada la importancia del tema de la sostenibilidad, la OMT incluye en la Agenda 2030 la meta 8.9 "Desarrollo Sostenible," la cual señala lo siguiente: "elaborar y poner en práctica políticas encaminadas a promover un turismo sostenible que cree puestos de trabajo y promueva la cultura y los productos locales". (p12)

En este sentido las directrices para el desarrollo sostenible del turismo y las prácticas de gestión sostenible se aplican a todas las formas de turismo en todos los tipos de destinos, incluidos el turismo de masas y los diversos segmentos turísticos. De modo que los principios de sostenibilidad se refieren a los aspectos medioambiental, económico y sociocultural del desarrollo turístico, habiéndose de establecer un equilibrio adecuado entre esas tres dimensiones para garantizar su sostenibilidad a largo plazo.

Por lo tanto, el turismo sostenible debe seguir los siguientes principios tal como se refieren en el siguiente esquema:

\begin{tabular}{|c|c|c|}
\hline 1 & $\begin{array}{l}\text { Dar un uso óptimo a los recursos } \\
\text { medioambientales, que son un elemento } \\
\text { fundamental del desarrollo turistico, } \\
\text { manteniendo los procesos ecológicos esenciales } \\
\text { y ayudando a conservar los recursos naturales y } \\
\text { la diversidad biológica }\end{array}$ \\
\hline PRINCIPIOS & $\begin{array}{l}\text { Respetar la autenticidad sociocultural de las } \\
\text { comunidades anfitrionas, conservar sus } \\
\text { activos culturales y arquitectónicos además } \\
\text { de sus valores tradicionales, y contribuir al } \\
\text { entendimiento y la tolerancia intercultural. }\end{array}$ \\
\hline DE & $\begin{array}{l}\text { Asegurar unas actividades económicas viables a } \\
\text { largo plazo, que reporten a todos los agentes } \\
\text { unos beneficios socioeconómicos bien } \\
\text { distribuidos entre los que se cuenten } \\
\text { oportunidades de empleo estable y de obtención } \\
\text { de ingresos y servicios sociales para las } \\
\text { comunidades anfitrionas, y que contribuyan a la } \\
\text { reducción de la pobreza }\end{array}$ \\
\hline
\end{tabular}

Tomado de la Organización Mundial del Turismo (2005) con adaptaciones de los autores 
Por otros lados en la Conferencia Globe 90, Tourism Stream, Action Strategy, de Vancouver, Canadá, se establecen los principios que orientan el turismo sostenible:

1. El turismo sostenible estimula la compresión de los impactos sobre los entornos naturales, culturales y humanos.

2. El turismo sostenible garantiza una distribución justa de costos y beneficios.

3. El turismo genera empleo local, tanto directamente en el sector turístico como en diversos sectores de apoyo y de gestión de recursos.

4. El turismo estimula industrias nacionales rentables: hoteles y otras instalaciones de alojamiento, restaurantes y otros servicios de alimentación, sistemas de transporte, artesanía y servicios de guía.

5. El turismo genera divisas para el país e inyecta capital y fondos frescos en le economía local.

6. El turismo diversifica la economía local, especialmente en zonas rurales donde el empleo agrícola puede ser esporádico o insuficiente.

7. El turismo sostenible requiere la adopción de decisiones en todos los sectores sociales, incluidos las poblaciones locales, de forma que el turista y los otros usuarios de los recursos puedan coexistir.

8. El turismo estimula mejoras en el transporte, las telecomunicaciones y otras infraestructuras comunitarias básicas.

9. El turismo crea instalaciones de esparcimiento que pueden ser usadas por las comunidades locales o por los visitantes nacionales extranjeros. También estimula y contribuye a costear la conservación de los yacimientos arqueológicos, edificios y barrios históricos.

10. El turismo estimula el uso productivo de terrenos marginales para la agricultura, permitiendo que grandes extensiones sigan cubiertas de su vegetación natal.

11. El turismo refuerza la autoestima comunitaria y ofrece la oportunidad de un mejor entendimiento y comunicación entre pueblos de diferente base cultural.

12. El turismo sostenible, desde el punto de vista del medio ambiente, pone de relieve la importancia de los recursos naturales y culturales para el bienestar económico y social de una comunidad y puede contribuir a preservarlos.

13. El turismo sostenible vigila, evalúa y gestiona los impactos del, desarrollo métodos fiables de seguimiento ambiental y contrarresta cualquier efecto negativo. ("The Internacional 
Ecoturism Society. 2004:35)

Desde esta perspectiva, se aprecia que el turismo sostenible es crucial para el desarrollo, no solo porque encierra el potencial de contribuir al crecimiento económico, sino que también puede ejercer una influencia positiva en las comunidades locales; preservar el patrimonio, la biodiversidad y los recursos naturales; promover el uso sostenible de los ecosistemas terrestres y marinos; y contribuir a la respuesta mundial al cambio climático, además que puede reducir las desigualdades y promover valores de comprensión cultural, tolerancia y paz (UNESCO 2017)

Con base a todo lo expresado, es fundamental que los sistemas educativos a nivel mundial se hagan presente ante las demandas ambientales y realicen propuestas viables de planes y programas curriculares que aporten al turismo sustentable, considerando que su concepto no se reduce solamente a parámetros económicos, sino que tiene un mayor alcance que va desde la preservación y conservación de la biodiversidad y recursos naturales hasta la promoción de los valores, dado que la tarea de la educación en la sociedad en palabras de Laudadío (2015), es aportar a la formación de los ciudadanos no solo para dar respuesta a un campo laboral competitivo, sino también en la formación de principios y valores cívicos y morales y ambientales, de allí que su contribución favorecerían no a una localidad en específico sino al mundo natural entero.

Asimismo, además de ser un derecho vinculado al desarrollo pleno de las personas, y ser el motor que genera progreso a las naciones, la educación incide decisivamente en las oportunidades y la calidad de vida de los individuos, las familias y las colectividades, por lo tanto, es un factor importante para el turismo sustentable, puesto que ayudaría a la formación de un ser humano con sentido de responsabilidad y conciencia clara hacia la conservación, de las riquezas naturales, y al patrimonio cultural.

Sabemos que la educación tiene carácter de modificar la sociedad, y brindar soluciones oportunas para resolver diferentes tipos de problemas que existen en la humanidad, por lo tanto no cabe duda que la educación para garantizar un turismo sostenible tiene un gran papel que desempeñar para la formación ciudadana, dado que proporcionaría todo los conocimientos adecuados sobre el funcionamiento del medio ambiente natural y sobre la manera en que los seres humanos pueden abordar el comportamiento y los ecosistemas para lograr la sostenibilidad. 
Con respecto a lo referido, Novo (2006) índica lo siguiente: El Decenio de las Naciones Unidas para la educación con miras al desarrollo sostenible pretende promover la educación como fundamento de una sociedad más viable para la humanidad e integrar el desarrollo sostenible en el sistema de enseñanza escolar a todos los niveles. El Decenio intensificará igualmente la cooperación internacional en favor de la elaboración y de la puesta en común de prácticas, políticas y programas innovadores de educación que aporten para el turismo sostenible.

De allí que podemos ver a la educación como la panacea que persigue la formación de conciencia no solo a los escolares si no a la ciudadanía en general para que, una vez que se tengan el conocimiento de todo lo que nos favorece el turismo sustentable podamos ser partícipes activos en la conservación de nuestro patrimonio, la biodiversidad y los recursos naturales; y promover el uso sostenible de los distintos ecosistemas.

\section{La Educación Ambiental: Base Para el Turismo Sostenible}

La Educación ambiental tiene como propósito, facilitar de forma didáctica la comprensión del mundo que nos rodea, además de crear conciencia sobre la responsabilidad que tiene cada ciudadano de su preservación, en palabras de Valera y Silva (2012) la educación ambiental se refiere a un "proceso permanente de carácter interdisciplinario, destinado a la formación, cuyas principales características son el reconocimiento de los valores, desarrollo de conceptos, habilidades y actitudes necesarias para una convivencia armónica entre seres humanos, su cultura y su medio biofísico circundante" (p. 196).

En referencia a lo expresado Fernández (s/f) considera que la educación ambiental debe fomentar el cambio social a partir del desarrollo de valores, actitudes y habilidades para asumir una responsabilidad ambiental. En este mismo sentido señala el autor, que la educación ambiental es una herramienta para mejorar las relaciones de los seres humanos con su medio y, por lo tanto, debe incidir en la prevención y resolución de problemas ambientales.

Con base a todo lo señalado, es importante mencionar que la educación ambiental no solo debe ser responsabilidad de los sistemas educativos formales, todos los organismos gubernamentales públicos o privados y medios de comunicación, están llamadas a contribuir y a promover sin mediación pedagógica explicita y de manera continua la formación ciudadana sobre la corresponsabilidad de todos con el medio ambiente, sin obviar que esta formación debe iniciarse desde las edades más tempéranos en los centros educativos formales. 
De allí que se puede lograr que desde la infancia se obtengan valores ambientales sólidos y por ende se conseguirá un adulto más responsable de su espacio natural inmediato. Desde esta misma perspectiva Fernandez (s/f) comenta que la educación ambiental (tanto formal como no formal) es, por sobre todas las cosas, una educación para la acción, desde una aproximación global e interdisciplinar, facilitando así un mejor conocimiento de los procesos ecológicos, económicos, sociales y culturales.

Es por ello que la educación ambiental debe fomentar el cambio social a partir del desarrollo de valores, actitudes y habilidades para asumir una responsabilidad ambiental; debe asumirse como una herramienta para mejorar las relaciones de los seres humanos con su medio y, por lo tanto, debe incidir en la prevención y resolución de problemas ambientales.

En este sentido, la UNESCO y el Programa de las Naciones Unidas para el Medio Ambiente, (Citados por Orgaz (2018) consideran los siguientes objetivos que debe seguir la educación ambiental:

- Responsabilizar y concienciar a las personas sobre los conocimientos del medio ambiente y sus problemáticas.

- Involucrar a las personas en las realidades, prácticas y experiencias de los problemas del medio ambiente que se perciben en sus territorios.

- Realizar actitudes que ayuden a las comunidades a fortalecer sus sentimientos de conservación y respecto de la naturaleza y el medio ambiente, así como su propia cultura.

- Desarrollar habilidades que fomenten la búsqueda de soluciones a las problemáticas ambientales actuales y prevengan los que puedan aparecer en el futuro.

- Fomentar acciones individuales o colectivas que corrijan o eviten problemas medio ambientales.

Desde el plano curricular, como materia de formación humana y ciudadana, la educación ambiental debe contemplar una serie de principios referidos en la siguiente tabla:

\begin{tabular}{|l|}
\hline Es considerada como un proceso continuado. \\
\hline Está integrada por todas las materias del saber y corriente científica. \\
\hline $\begin{array}{l}\text { Debe fomentar la participación, prevención y el trabajo de búsqueda de } \\
\text { soluciones. }\end{array}$ \\
$\begin{array}{l}\text { Tiene como base el pensamiento crítico e innovador en cualquier tiempo } \\
\text { y lugar. }\end{array}$ \\
\hline Es individual o colectiva. \\
\hline $\begin{array}{l}\text { Pretende formar ciudadanos con conciencia local y global acerca de las } \\
\text { problemáticas medio ambientales que nos rodean. }\end{array}$ \\
\hline Es un derecho de todas las personas. \\
\hline Debe ser ejercida por todos los ciudadanos. \\
\hline Es holística. \\
\hline
\end{tabular}




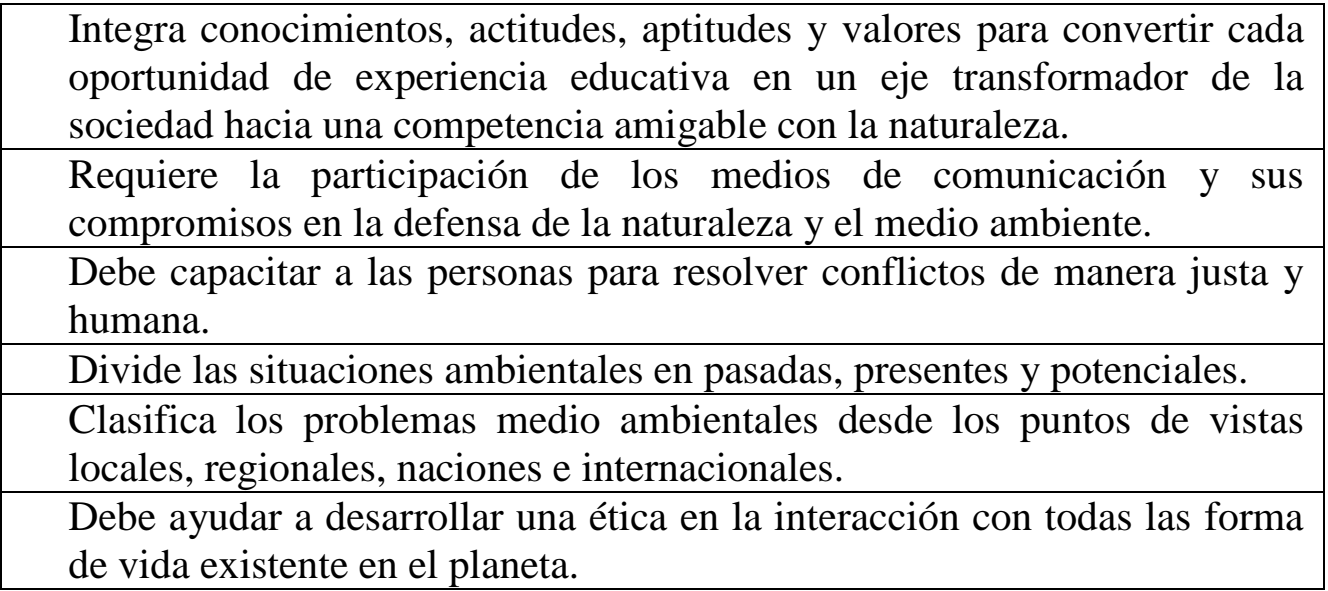

Tomados de Valera y Silva (2012)

Al analizar todo estos aspectos, se puede apreciar que la incorporación de la educación ambiental para promover el turismo sustentable, es fundamental, dado a que por ser parte de la formación de valores ciudadano se generaría un cambio en la actitud de la personas y por ende esto restaría los impactos negativos hacia el medio ambiente, hacia las riquezas y patrimionios culturales; considerando que el desafío de la educación ambiental en palabras de Ramos y Fernández (2013) “es promover una nueva relación de la sociedad humana con su entorno, a fin de procurar a las generaciones actuales y futuras un desarrollo personal y colectivo más justo, equitativo y sostenible"( p.295).

De allí que en palabras del mismo autor, la educación ambiental debe fomentar el cambio social a partir del desarrollo de valores, actitudes y habilidades para asumir una responsabilidad ambiental, mientras que el turismo puede convertirse en un disparador de situaciones que se pueden materializar en "buenas prácticas" (control de la producción de residuos y del consumo de agua, energía, conservación de espacios naturales, etc.) o nuevas demandas que obliguen al mercado a tomar medidas de control.

\section{Manta un lugar del Ecuador para la Educación ambiental y el turismo sustentable}

Es importante destacar que Ecuador se encuentra entre los 17 países con mayor diversidad natural y cultural del planeta, constituido por las costas del Pacífico, con sus maravillosas playas; la cordillera andina con sus volcanes de más de 5.000 metros de altura; las Islas Galápagos, con sus playas de arena blanca y su flora y fauna únicas, y la mágica Amazonía, con sus inmensas selvas. Además posee una gran diversidad cultural con alrededor de 30 nacionalidades, pueblos indígenas, afroecuatorianos y pueblos en aislamiento voluntario que forman un país pluricultural y multiétnico. 
Se aprecia así la riqueza del país en materia turística, convirtiéndose ésta en generadora de una economía que favorece a gran parte de la población, además de posicionarlo como uno de los destinos más cotizados a nivel mundial.

Una de las zonas con alto potencial turístico del país está ubicada en la provincia de Manabí, es la ciudad de Manta, también conocida como el puerto de la brisa y la imaginación se caracteriza por sus aguas profundas con playas hermosas, diversidad de ecosistemas y riquezas culturales; es el puerto mayor turístico, marítimo y pesquero del Ecuador, y ha sido declarada como la principal zona turística del país. Además de ser el puerto más importante; su dinamismo se expresa por su gran desarrollado en infraestructuras hoteleras y alta producción de atún café tagua cacao y aceite vegetales los cuales son muy cotizados en el mercado mundial.

Tomando en consideración el alto potencial turístico del Ecuador, existe una gran preocupación por su biodiversidad, una necesidad de protegerla y al mismo tiempo generar un equilibrio en lo social a través de la conservación y del manejo consciente de los recursos naturales. Carrillo (2017)

El turismo es de las pocas actividades productivas en las que se pueden obtener ingresos, beneficiar a las comunidades y al mismo tiempo conservar el medio ambiente. Entonces, es el turismo sostenible la opción que tiene el Ecuador para convertirse en potencia turística. Sin embargo señala el autor antes citado que el turismo sustentable ha dejado de ser solo una alternativa que puede ser tomada o dejada de lado por las empresas turísticas, hoy es una obligación. Las características del país deben ser bien aprovechadas por los gobiernos a la hora de diseñar políticas en esta materia. Un manejo consciente de la biodiversidad permitirá crear una marca país mucho más competitiva y atractiva para atraer turistas. (Carrillo 2017)

Es necesario que el turismo sostenible para el país sea una prioridad y no un gasto. Las propuestas deben integrar a todos los actores de una sociedad e incluir variables de integridad ambiental, justicia social y rentabilidad económica, asegurando con esto la sostenibilidad de las actividades productivas del país en el mediano y largo plazo. Esta estrategia permitirá reducir los costos, aumentar el branding de las empresas y generar confianza entre el consumidor y el operador.

Con base a lo expresado, es importante que las políticas socioeconómicas que asume la nación no se tome al turismo solo para estimular las industrias nacionales rentables, la creación 
de hoteles y otras instalaciones de alojamiento, restaurantes y servicios de alimentación, sistemas de transporte, para generar empleo, sino que se asumen medidas con base en la educación ambiental, bien pensadas, definidas en pro a la formación de los ciudadanos que así como aporta para la actividad económica y social, contribuya para la vida en armonía con el medio ambiente y el patrimonio cultural.

Este llamado reiterativo de la incorporación de la educación ambiental para un turismo sostenible obedece significativamente por la falta de cultura ambiental que existe tanto en los lugareños como a los visitantes que arriban a la ciudad de Manta, se observa con preocupación la disposición final de los desechos sólidos en zonas que perjudican al ecosistema terrestre y al marítimo, la construcción desmesurada que resta cada vez los espacios naturales y a la propagación de la informalidad del comercio de forma clandestina. Esta situación demanda una posición activa y eficiente del estado y mayor presencia de la educación la ciencia la tecnología, la industria y el comercio, porque todos son corresponsables del cuidado y de generar alternativas para la preservación de las reservas naturales.

\section{Consideraciones finales}

Es imperativo que se asuma un currículo educativo contextualizado, que incluya la educación ambiental como la herramienta para impulsar el turismo sostenible, donde los docentes desarrollen estrategias con mayores niveles de concreción y con una postura socio crítica más cónsona con las demandas sociales y ambientales, en el que se implemente como lo señala Cazares, Aranguren y Moncada (2017) proyectos de investigación y desarrollo con elemento de transformación social de la realidad local inmediata, que permite un aprendizaje más significativo y pertinente a la propuestas educativa para promover una educación turística dirigida asegurar la sustentabilidad de las reservas ecológicas y al conocimiento integral de la reserva sobre la importancia del manejo sustentable de sus recursos en la actividad turística.

Además es necesario que se desarrollen programas descentralizados con un enfoque ambientalista con soportes conceptuales y metodologías sólidas que permiten diseñar ejecutar políticas públicas educativas que garanticen calidad, y el desarrollo de oportunidades de aprendizaje en el ámbito de la educación ambiental para consolidar la cultura del turismo sostenible para ciudad de Manta. 


\section{REFERENCIAS}

Carrillo, R. (2017) El turismo sustentable es la opción que tiene el Ecuador para convertirse en potencia turística Universidad Internacional del Ecuador. Disponible en: https://dialoguemos.ec/2017/09/el-turismo-sustentable-es-la-opcion-que-tiene-elecuador-para-convertirse-en-potencia-turistica/

Cazares, P. Aranguren, J y Moncada. A (2017) Educación para el turismo sustentable en la escuela ecuatoriana. Una propuesta para la Parroquia La Libertad, Cantón Espejo, Provincia del Carchi. Revista de Investigación, vol. 41, núm. 92, septiembre-diciembre, 2017, pp. 34-55 Universidad Pedagógica Experimental Libertador Caracas, Venezuela

Conferencia de la Organización de Naciones Unidas sobre Desarrollo Sostenible. (1999). Presented at the Séptima sesión del comité de Turismo Sostenible.

Conferencia de la Organización de Naciones Unidas sobre Medio Ambiente y Desarrollo. (1992). Presented at the Cumbre de la Tierra, Brasil.

Development. Vancouver BC, Canada

Fernández, G.(s/f) Postítulo en Educación para un Desarrollo Sustentable (e/c). UNR. Argentina. Disponible: http://revistaea.org/pf.php?idartigo=164

GLOBE (1990). ' 90 Conference, Tourism Stream, Action Strategy for Sustainable Tourism

Laudadío, J. (2015). Ser profesor universitario, desafío digno de ser. Perspectiva Educacional. Formación de Profesores.

Novo, M. (2006a). El desarrollo sostenible. Su dimensión ambiental y educativa. Madrid: UNESCO-Pearson.

Organización de las Naciones Unidas para la Educación la Ciencia y la Cultura UNESCO (2017) Turismo - entre la oportunidad y la amenaza

Organización Mundial del Turismo. Agenda para planificadores locales: Turismo sostenible y gestión municipal. Edición para América Latina y el Caribe. 1999.

Orgaz-Agüera F. (2018): "Educación ambiental: concepto, origen e importancia. El caso de República Dominicana ", Revista DELOS Desarrollo Local Sostenible n. 31(febrero 2018). En línea: https://www.eumed.net/rev/delos/31/francisco-orgaz.html //hdl.handle.net/20.500.11763/delos31francisco-orga

Ramos, G. y Fernandez, G. ( 2013) La educación ambiental: un instrumento para el turismo sustentable. Revista Hospitalidade. São Paulo, v. X, n. 2, p. 296 - 312, dez. 2013

Red de Periodistas por el Desarrollo Sostenible (RPDS) (2018) LA IMPORTANCIA DE PROMOCIONAR EL TURISMO SOSTENIBLE disponible en https://www.comunicacionsostenible.co/site/la-importancia-de-promocionar-el-turismosostenible/

Troncoso, B. (2012). Propuesta para una política de desarrollo turístico sostenible. Retrieved from http://kiskeya-alternative.org/publica/bolivar/desarrs.html

Valera Mejía, F. y Silva Naranjo, E. (2012). Guía de capacitación en educación ambiental y cambio climático. USAID, CDCT y The Nature Conservancy: Santo Domingo. 